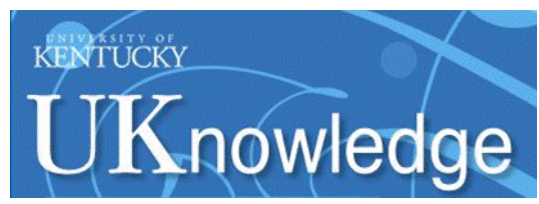

University of Kentucky

UKnowledge

\title{
6-1-1994
}

\section{A Protostellar Jet Model for the Water Masers in W49N}

\author{
Mordecai-Mark Mac Low \\ University of Chicago \\ Moshe Elitzur \\ University of Kentucky, moshe@pa.uky.edu \\ James M. Stone \\ University of Maryland - College Park \\ Arieh Königl \\ University of Chicago
}

Follow this and additional works at: https://uknowledge.uky.edu/physastron_facpub

Part of the Astrophysics and Astronomy Commons, and the Physics Commons

Right click to open a feedback form in a new tab to let us know how this document benefits you.

\section{Repository Citation}

Low, Mordecai-Mark Mac; Elitzur, Moshe; Stone, James M.; and Königl, Arieh, "A Protostellar Jet Model for the Water Masers in W49N" (1994). Physics and Astronomy Faculty Publications. 225.

https://uknowledge.uky.edu/physastron_facpub/225

This Article is brought to you for free and open access by the Physics and Astronomy at UKnowledge. It has been accepted for inclusion in Physics and Astronomy Faculty Publications by an authorized administrator of UKnowledge. For more information, please contact UKnowledge@lsv.uky.edu. 


\section{A Protostellar Jet Model for the Water Masers in W49N}

Digital Object Identifier (DOI)

http://dx.doi.org/10.1086/174196

\section{Notes/Citation Information}

Published in The Astrophysical Journal, v. 427, no. 2, p. 914-918.

(c) 1994. The American Astronomical Society. All rights reserved.

The copyright holder has granted permission for posting the article here. 


\title{
A PROTOSTELLAR JET MODEL FOR THE WATER MASERS IN W49N
}

\author{
Mordecai-Mark Mac Low, ${ }^{1}$ Moshe Elitzur, ${ }^{2}$ James M. Stone, ${ }^{3}$ AND Arieh KöNiGL ${ }^{1}$ \\ Received 1993 October 29; accepted 1993 November 29
}

\begin{abstract}
Observations by Gwinn, Moran, \& Reid of the proper motions of water masers in W49N show that they have an elongated distribution expanding from a common center. Features with high space velocity only occur far from the center, while low-velocity features occur at all distances. We propose that these observations can be interpreted in terms of a shell of shocked molecular gas that is driven by the expanding cocoon of a high-velocity protostellar jet. We present three-dimensional numerical simulations in support of this interpretation and argue that this source provides a unique opportunity for a detailed study of jet-driven cocoons.

Subject headings: ISM: individual (W49) - ISM: jets and outflows - masers - stars: formation
\end{abstract}

\section{INTRODUCTION}

Strong water maser emission is an important signpost of the star formation process. The maser action is triggered by powerful outflows (e.g., Elitzur, Hollenbach, \& McKee 1989), a characteristic property of newly formed stars that manifests itself in emission by a number of species. Among the various outflow diagnostics, extreme brightness distinguishes water masers as unique probes of very detailed structure, not accessible by other means.

Proper motion measurements of the water masers in $\mathrm{W} 49 \mathrm{~N}$ (more properly called W49A North, as in Welch et al. 1987) by Gwinn, Moran, \& Reid (1992) reveal an intriguing velocity field with a spatial structure suggestive of an expansion from a common center. The expansion speed is almost constant, $v \approx 20 \mathrm{~km} \mathrm{~s}^{-1}$, over the range $R \approx 3 \times 10^{16}-10^{17} \mathrm{~cm}$, where $R$ is the distance from the center. At $\sim 10^{17} \mathrm{~cm}$ the $v$ versus $R$ curve rises sharply and becomes nearly vertical, with the expansion speed reaching a maximum $\sim 200 \mathrm{~km} \mathrm{~s}^{-1}$ at $R \approx 2 \times 10^{17} \mathrm{~cm}$. If individual maser features are interpreted as gas condensations ("bullets") that move out from a common center, then each condensation must travel at a uniform speed of $\sim 20 \mathrm{~km} \mathrm{~s}^{-1}$ through the interior of the region and undergo a rapid acceleration to $\sim 200 \mathrm{~km} \mathrm{~s}^{-1}$ at its edge. This was the interpretation adopted by Gwinn et al. (1992), who listed a number of possible mechanisms that could be responsible for the sudden acceleration at $\sim 10^{17} \mathrm{~cm}$.

In contrast, Mac Low \& Elitzur (1992, hereafter Paper I) suggested that the masers are surface phenomena that trace the envelope of an expanding bubble. In this picture, the observed $v$ versus $R$ curve implies that the shell has an elongated shape and is expanding rapidly (at a speed $\sim 200 \mathrm{~km} \mathrm{~s}^{-1}$ ) along the axis and slowly (at a speed $\sim 20 \mathrm{~km} \mathrm{~s}^{-1}$ ) perpendicular to it. Subsequent data analysis by Gwinn (1993) has verified that this model accurately reproduces the observations. Paper I also considered the possible dynamical origin of the observed mor-

\footnotetext{
${ }^{1}$ Department of Astronomy and Astrophysics, University of Chicago, 5640 South Ellis Avenue, Chicago, IL 60637; mordecai@jets.uchicago.edu, arieh@jets.uchicago.edu.

${ }_{2}$ Department of Physics and Astronomy, University of Kentucky at Lexington, Lexington, KY 40506-0055; moshe@ukcc.uky.edu

${ }_{3}$ Department of Astronomy, University of Maryland at College Park, College Park, MD 20742; jstone@astro.umd.edu.
}

phology. It was suggested that the elongated shell corresponds to an interstellar bubble that is driven by a high-velocity $\left(\sim 1500 \mathrm{~km} \mathrm{~s}^{-1}\right)$, spherically symmetric stellar wind that expands into a medium with an anisotropic density distribution. A detailed calculation showed that a spherical outflow expanding into a cylindrical cavity sweeps up the ambient gas into an elongated shell whose evolution is consistent with the measured $v$ versus $R$ curve. However, the postulated density distribution, with a newly formed, massive star located on the symmetry axis, was admittedly ad hoc and was used merely to illustrate the proposed new kinematic interpretation of the W49N masers in the context of a spherically symmetric stellar wind model.

In this paper we propose a more plausible origin for the elongated shell in W49N. We suggest that the outflow itself is anisotropic, specifically that it is a protostellar jet, and we demonstrate that the motion of the shell of swept-up gas that is driven out by these jets can reproduce the observed $v$ versus $R$ curve even when the ambient medium is isotropic. The morphology of bubbles evacuated by high-velocity jets was first analyzed by Scheuer (1974) in the context of extragalactic radio sources. He showed that the momentum transferred to the ambient medium at the jet head, where the outflow is shocked and decelerated, causes the bubble to elongate in the direction of the jet propagation. He pointed out, however, that the bubble also expands (albeit at a lower speed) in the direction normal to the jet axis on account of the high pressure of the "cocoon" of hot jet material that envelopes the jets. As was demonstrated by subsequent numerical experiments (e.g., Norman et al. 1982), the formation of an extensive cocoon requires a high Mach-number jet whose density is lower than that of the ambient gas. In the case of protostellar jets, it is also necessary to verify that the outflow speed is high enough, and its density low enough for the shocked gas not to cool before flowing out of the jet-head region (e.g., Blondin, Fryxell, \& Königl 1990). The structure and evolution of jet cocoons have been extensively investigated in recent years, both by analytic approximations and by numerical modeling (e.g., Begelman \& Cioffi 1989; Cioffi \& Blondin 1992; Loken et al. 1992).

The basic kinematic property of jet-driven cocoons-a rapid axial elongation coupled with a slower transverse expansionis precisely the behavior invoked in Paper I to account for the $\mathrm{H}_{2} \mathrm{O}$ maser motions in W49N. We now proceed to substan- 
tiate this scenario with the help of detailed dynamical calculations $(\$ 2)$. We discuss the general applicability of this model in $\S 3$, which also contains our conclusions.

\section{JET-POWERED COCOON EXPANSION}

In this section we first present an approximate analytic description of the dynamics of an adiabatic cocoon and give the condition for the jet termination shock to be nonradiative. This condition must be satisfied if a hot, high-pressure cocoon is to develop. On the other hand, if the shell of swept-up gas that marks the boundary of the cocoon is to be dense enough for maser emission, the outer shocks that are driven into the ambient medium must be radiative. We show that the requirements on the termination shock and the outer shocks can plausibly be satisfied simultaneously. Next, we examine the evolution of the cocoon numerically, using a three-dimensional hydrodynamical code that includes radiative cooling. We demonstrate that the $v$ versus $R$ curve for the dense shell can reproduce the observations of $\mathrm{W} 49 \mathrm{~N}$.

\subsection{Dynamics}

Consider the collimated jet sketched in Figure 1 with radius $r_{j}$, velocity $v_{j}$, density $\rho_{j}$, and mechanical luminosity

$$
L_{j}=\frac{\pi}{2} r_{j}^{2} \rho_{j} v_{j}^{3}
$$

moving into a uniform ambient medium of density $\rho_{a}$ and negligible pressure. The jet terminates in a shock, often referred to as the "Mach disk." If the termination shock is adiabatic and the density ratio $\eta \equiv \rho_{j} / \rho_{a} \leq 1$, the hot, shocked jet gas fills a cocoon of volume $V_{c}$ that envelopes the jet. The jet termination shock is preceded by a bow shock that propagates into the ambient medium. On the sides of the jet, the high-pressure cocoon also drives shocks that, together with the bow shock, sweep the ambient gas up into a thin shell. The cocoon typically expands rapidly along the direction of jet propagation ( $z$-axis), elongating at the advance speed $\dot{z}=v_{h}$ of the jet head, and more slowly (at $\dot{r}=v_{c}$ ) in the transverse direction ( $r$-axis). In the nomenclature of interstellar bubbles, the tips of the cocoon are "momentum driven," while the sides are "energy driven" (cf. Masson \& Chernin 1993).

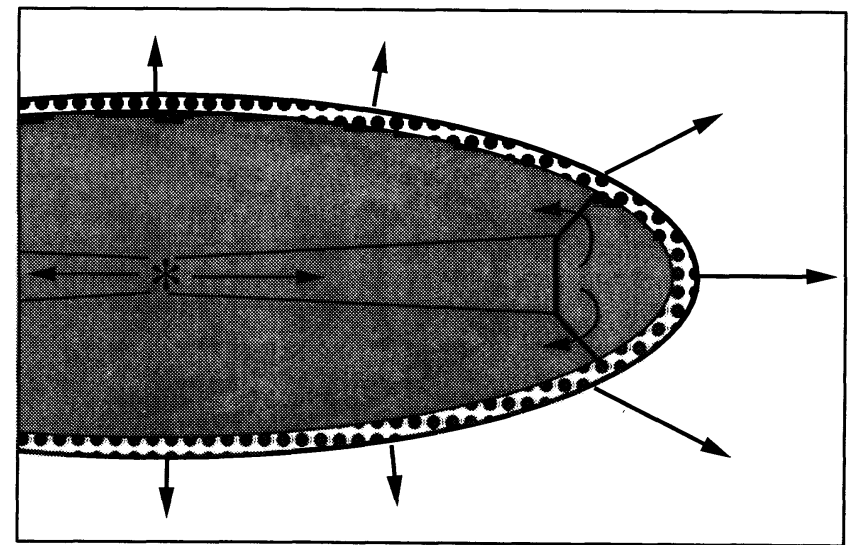

Fig. 1.-Structure of our proposed model for water masers. A protostellar jet gets stopped and heated at a termination shock. The resulting hot gas sweeps up a shell from the ambient gas. The jet termination shock and the outer shock, in which the masers form, are shown as thicker lines. The ends move faster than the sides, producing higher velocities at larger radii, as observed in W49N.
The value of $v_{h}$ can be found by balancing, in the frame of the jet head, the jet thrust $\pi r_{j}^{2} \rho_{j}\left(v_{j}-v_{h}\right)^{2}$ and the ram pressure $\rho_{a} v_{h}^{2} A_{h}$ of the ambient medium; here $A_{h}$ is the cross-sectional area of the jet head, which can, in principle, exceed the cross sectional area of the jet. In the limit $\eta \ll 1$ that is of interest to us, $v_{h}$ is $\ll v_{j}$ and one gets

$$
v_{h} \approx\left(\frac{2 L_{j}}{v_{j} A_{h} \rho_{a}}\right)^{1 / 2},
$$

which, for $A_{h} \approx \pi r_{j}^{2}$, reduces to

$$
v_{h} \approx v_{j}\left(\rho_{j} / \rho_{a}\right)^{1 / 2} .
$$

Integrating equation (2) and allowing the jet head area to vary with time according to $A_{h}=A_{h 0} t^{\alpha}$, gives the length of the jet as

$$
z=\frac{2}{2-\alpha}\left(\frac{2 L_{j}}{v_{j} A_{h 0} \rho_{a}}\right)^{1 / 2} t^{1-\alpha / 2} .
$$

The maximum width $r$ of the cocoon can be roughly estimated by taking the internal pressure to be $p \approx L_{j} t / V_{c}$, which ignores the adiabatic expansion losses. Balancing the internal pressure against the ram pressure $\rho_{a} v_{c}^{2}$ on the sides of the cocoon, yields

$$
r^{2} \dot{r}^{2}=L_{j} t / \pi \rho_{a} z
$$

In deriving this expression, we have neglected the effect of the ambient magnetic field. (We discuss this further in $\S 2.2$ ). Integrating equation (5) and substituting from equation (4), one obtains

$$
r=\text { const } \times\left(L_{j} v_{j} A_{h 0} / \rho_{a}\right)^{1 / 8} t^{(4+\alpha) / 8} .
$$

Equations (4) and (5) imply

$$
\frac{\dot{z} / z}{\dot{r} / r}=\frac{8-4 \alpha}{4+\alpha},
$$

which shows that the axial expansion of a jet-driven bubble in a uniform ambient medium will always exceed the transverse expansion if $\alpha<0.8$. The applicable value of $\alpha$ depends on the details of the flow at the jet head. In the original formulation of Scheuer (1974), the solid angle subtended by the head was taken to be constant, corresponding to $\alpha=1$. In contrast, Begelman \& Cioffi (1989) considered the limit $\alpha=0$ where the head size remains fixed as the bubble expands. The actual growth of the head depends on the details of the flow at the working surface. If the W49N masers trace the boundary of a cocoon, then $\dot{z} / \dot{r} \approx 10$ and $z / r \approx 5-6$ (see Fig. $2 a$ ), yielding $\alpha \approx 0-0.23$, consistent with the Begelman \& Cioffi (1989) assumption. The elongation of the bubble may actually be enhanced beyond the result for a homogeneous medium because the jet propagation direction probably coincides with the ambient density gradient, amplifying the axial expansion rate (e.g., Königl 1982).

We now consider the condition for the jet termination shock to be adiabatic. Roughly, this condition is that the cooling parameter $\chi \equiv d_{\text {cool }} / r_{j}$, where $d_{\text {cool }}$ is the cooling length behind the shock, be larger than 1 (e.g., Blondin et al. 1990; Stone \& Norman 1993a). An approximate relation for the cooling length behind a shock of speed $v_{s 7} \equiv v_{s} /\left(10^{7} \mathrm{~cm} \mathrm{~s}^{-1}\right)>2$, found numerically using the code described in $\S 2.2$, is

$$
d_{\text {cool }} \approx 2 \times 10^{16} v_{s 7}^{5} n^{-1} \mathrm{~cm},
$$




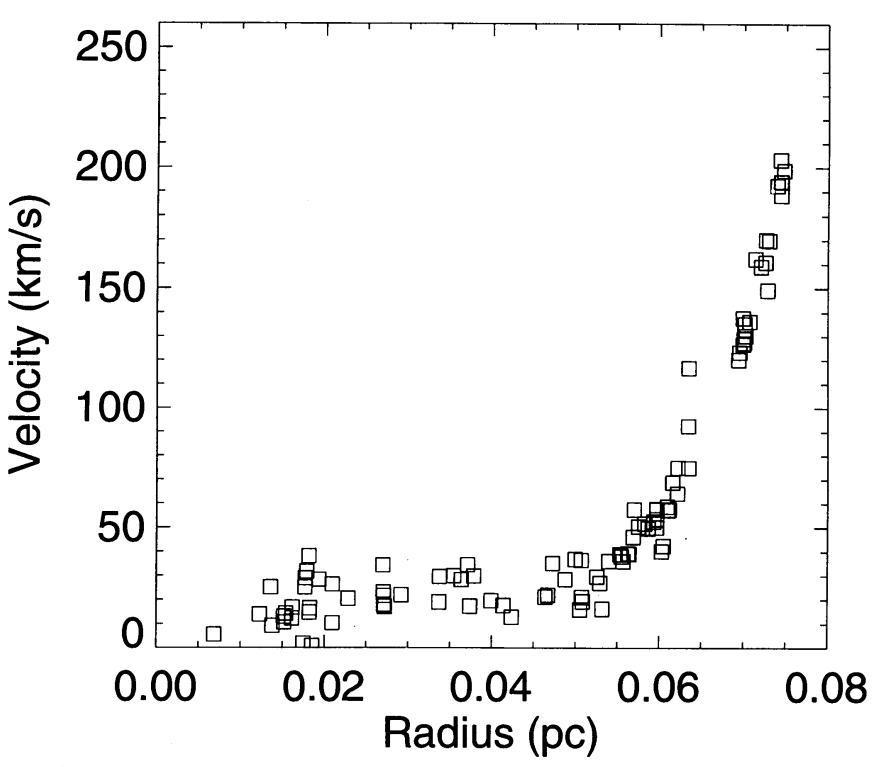

FIG. $2 a$

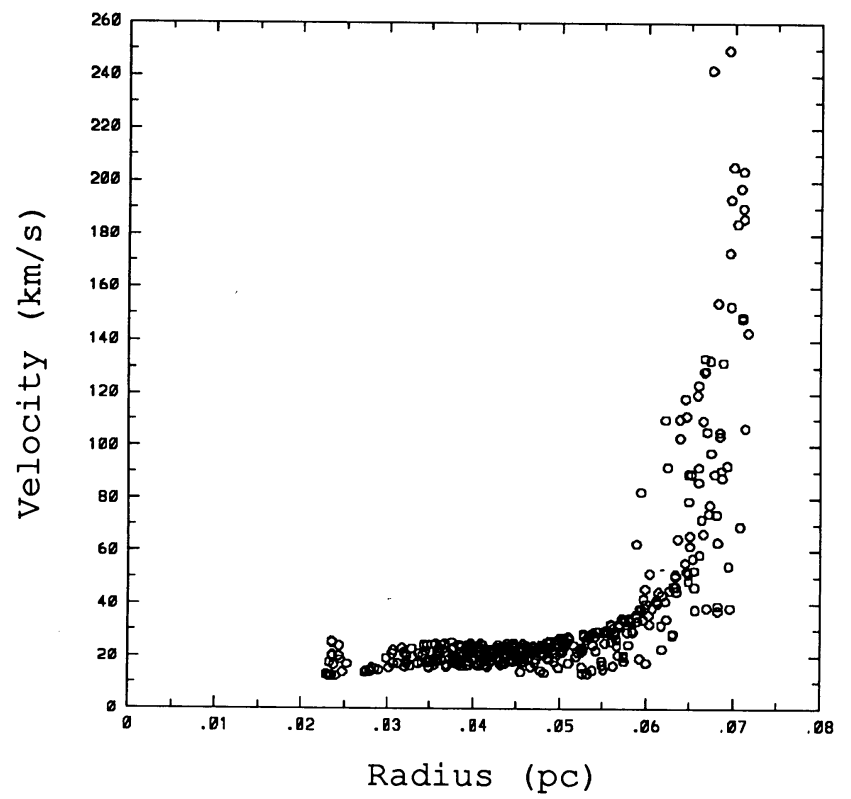

FIG. $2 b$

FIG. 2. - (a) Observed and $(b)$ computed space velocities and distances from the center of expansion in W49N. The observed maser spots shown are from Fig. 5 of Gwinn et al. (1992). The computed points are values for high-density zones in the numerical model.

where $n$ is the preshock number density. Using equation (2) to express the jet particle density in terms of the ambient number density $n_{a}$, we thus obtain

$$
\chi=20 v_{j 8}^{7} /\left(n_{a 6} r_{j 16} v_{h 7}^{2}\right),
$$

where $v_{j}, v_{h}, n_{a}$, and $r_{j}$ have been normalized by $10^{8} \mathrm{~cm} \mathrm{~s}^{-1}, 10^{7}$ $\mathrm{cm} \mathrm{s}^{-1}, 10^{6} \mathrm{~cm}^{-3}$, and $10^{16} \mathrm{~cm}$, respectively.

While the jet termination shock is adiabatic $(\chi>1)$, the outer shock all the way around the cocoon will be radiative, since the cooling parameter is a factor $\sim \eta^{3}$ smaller. The density behind this radiative shock will be far higher than the ambient density, so the entire shell of swept-up ambient gas will reach the high densities needed for maser emission.

\subsection{Numerics}

In order to actually reproduce the observed distribution of velocity versus radius shown in Figure $2 a$, we must turn to numerical computations. Recently, Stone \& Norman (1993a, b, 1994) have described a series of simulations of the two- and three-dimensional dynamics of nonadiabatic, protostellar jets. (Related work has been done by de Gouveia Dal Pino \& Benz 1993.) The simulations of Stone \& Norman use a threedimensional implementation of the piecewise parabolic method (PPM; Colella \& Woodward 1984). This high-order algorithm for gas dynamics does not require the use of an artificial viscosity to capture shocks, thus minimizing the numerical diffusion of sharp features in the flow. To model nonadiabatic jets, Stone \& Norman extend the PPM algorithm with a time-dependent, nonequilibrium, radiative cooling method. This method, which allows for ionization, recombination, and history-dependent cooling effects, has been shown to give a more accurate estimate of the total cooling rate than methods based on the assumption of complete ionization (Stone \& Norman 1993a).

Stone \& Norman model a jet propagating into a homogeneous medium in Cartesian coordinates. The jet is introduced as an inflow boundary condition with velocity $v_{j}$, density $\rho_{j}$, and temperature $T_{j}$, for $r<r_{j}$. For $r>r_{j}$, a reflecting bound- ary condition is used on the equatorial plane. Reflecting boundaries are also used on the two symmetry planes, so only one quadrant of the jet is computed. Outflow boundary conditions are used everywhere else. A grid of $80 \times 80 \times 480$ zones is used, with eight zones in a jet radius.

The limited resolution of these simulations prevents an accurate representation of the thin cold shell for ambient densities high enough to produce masers. If models using such densities are attempted, a small fraction of the mass of the cold shell cannot be prevented from diffusing into the hot pressurized interior, causing the hot gas to rapidly cool. Instead, we use the models presented in Stone \& Norman, which have been run at a much lower density, and then scale them to a higher ambient density. Figure 3 shows the computed density distribution of a jet with $n_{a}=n_{j}=65 \mathrm{~cm}^{-3}, v_{j}=200 \mathrm{~km} \mathrm{~s}^{-1}$, and $r_{j}=2.5$ $\times 10^{15} \mathrm{~cm}$, at a time of $320 \mathrm{yr}$. Using equation (9), we derive a cooling parameter $\chi=4$ for this jet.

There are three constraints that we wish to satisfy. First, the speed of the fastest observed masers cannot exceed that of the jet head, so $v_{h} \gtrsim 200 \mathrm{~km} \mathrm{~s}^{-1}$. Second, water masers can only form if the preshock density $n_{a} \gtrsim 3 \times 10^{6} \mathrm{~cm}^{-3}$ (Elitzur et al. 1989). Finally, the cooling parameter $\chi$ must remain the same in order to get the same cocoon structure. We proceed by requiring $\chi$ to remain constant in equation (9). Substituting in the required head velocity and preshock density, we find the jet velocity that will satisfy all three constraints, $v_{j} \approx 1100 r_{j 16}^{1 / 7}$ $\mathrm{km} \mathrm{s}^{-1}$. We can now use equation (3) to find the jet density, $n_{j} \approx 10^{5} r_{j 16}^{-2 / 7} \mathrm{~cm}^{-3}$, and hence the mass outflow rate in the two jets, $M_{j} \approx 10^{-4} r_{j 16}^{13 / 7} M_{\odot} \mathrm{yr}^{-1}$.

A jet with these parameters will have the same structure as the jet computed by Stone \& Norman, so we can compare the $v$ versus $R$ curve for this jet to the observations. We have scaled the jet radius to $1000 \mathrm{AU}$, but it could be much smaller without strongly affecting our results, due to the weak dependence of the inferred jet velocity and density on $r_{j}$. Whatever the details of the acceleration mechanism, protostellar jet velocities are of order the escape velocity at the origin of the outflow. Thus, the massive star at the center of the maser distribution (Welch et 


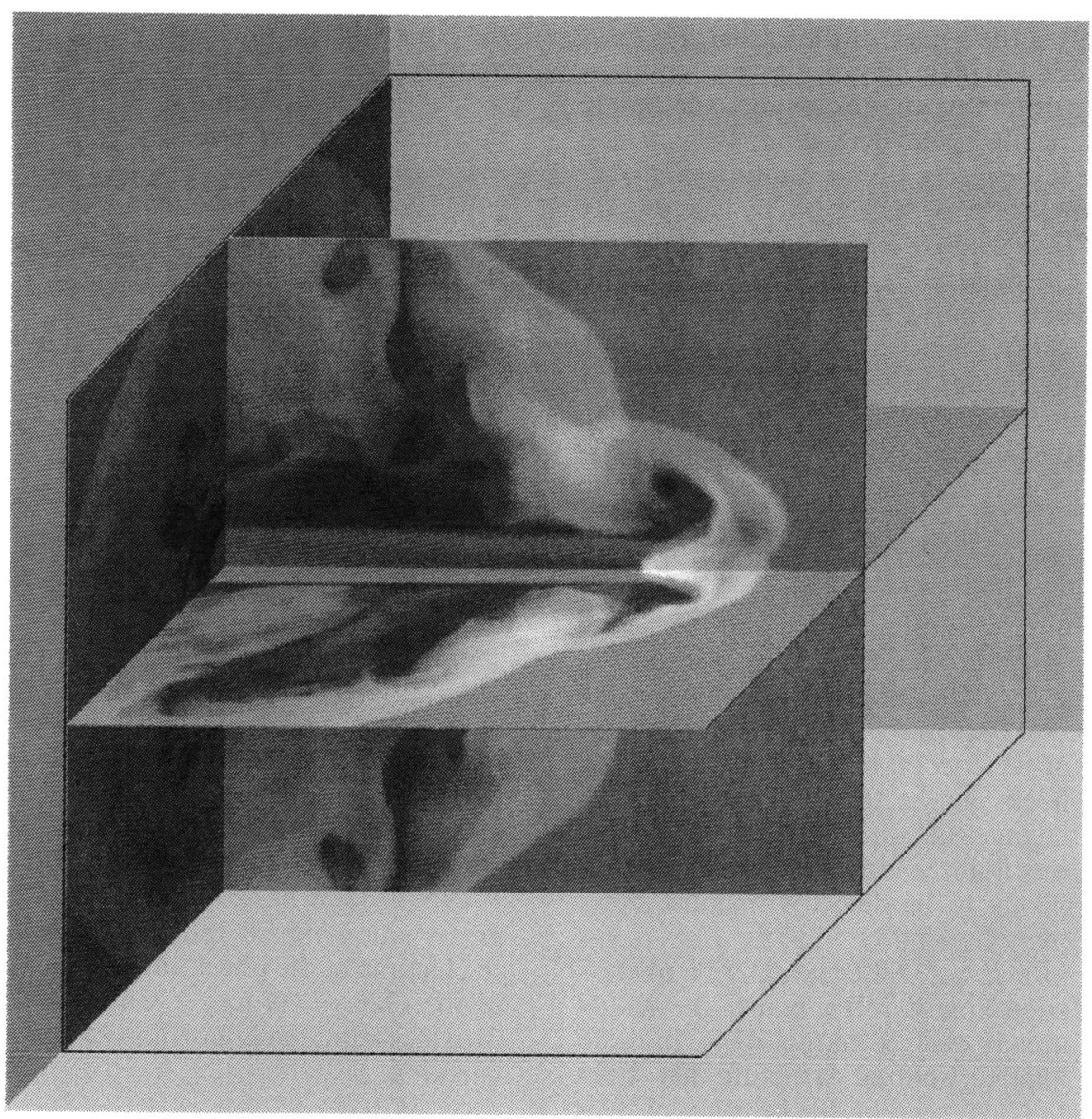

Fig. 3.-Density distribution of computed jet. The log of the density is shown, with black low and white high. Notice the density variations in the outer shell, which may correspond with clumps of masers. Only the outer third of the computed grid is shown.

al. 1987) could plausibly drive a $\sim 1000 \mathrm{~km} \mathrm{~s}^{-1}$ jet. Because of the high cost of each run, we were unable to optimize the fit to the observations. Nevertheless, Figure $2 b$ shows that with these parameters, the computed $v$ versus $R$ curve reproduces the observed curve well.

Each of the data points in Figure $2 b$ are zones in the numerical simulation in which the density exceeds some arbitrarily chosen large value. Only a randomly selected sample of such points are plotted to illustrate the shape of the curve. The scatter of the points is associated with this sampling and with the finite numerical resolution of the simulation; as more points are plotted the computed curve becomes continuous. We find that changing the numerical resolution of the simulation has little effect on the shape of the curve. Instead, the shape depends upon the physical parameters in the jet, for example the value of the cooling parameter $\chi$. It is also possible that the shape may be modified by considering flows which are not perfectly collimated, although we have not investigated this effect here.

Our neglect of the magnetic field in deriving jet properties can be checked. The ambient field strength required for magnetic stress to be in equipartition with the ram pressure of the cocoon is (Königl 1982)

$$
B_{\mathrm{eq}}=\left(32 \pi \rho_{a} v_{c}^{2} / 9\right)^{1 / 2}=20 \mathrm{mG},
$$

where we have used $n_{a}=3 \times 10^{6} \mathrm{~cm}^{-3}$ as described above, and taken $v_{c}=20 \mathrm{~km} \mathrm{~s}^{-1}$ from Figure $2 a$. By comparison,
Fiebig \& Güsten (1989) measured the magnetic field in the maser clumps behind the shock front and found a maximum strength of only $50 \mathrm{mG}$. Since the field in the clumps has been shock compressed, it greatly exceeds the ambient field, which thus must be much lower than $B_{\text {eq }}$. Therefore, to first order the effect of magnetic fields can be neglected.

We also want to call attention to another feature of Figure 3. The outer shell of the cocoon is subject to dynamical instabilities, including the Rayleigh-Taylor and Kelvin-Helmholtz instabilities and the Vishniac overstability (Vishniac 1983; Mac Low \& Norman 1993). Figure 3 shows that these instabilities lead to a complex network of clumps and filaments. These inhomogeneities produce the scatter in the computed curve shown in Figure $2 b$. Furthermore, Gwinn, Moran, \& Reid (1993) have found that the water masers are not evenly distributed, but rather that clusters of masers occur, with sizes of $2300 \mathrm{AU}$. Our computations are far too underresolved to make quantitative comparisons, but we can draw the qualitative conclusion that the computed instabilities could explain the observed clumping.

\section{DISCUSSION AND CONCLUSIONS}

Our proposal that the water masers in $\mathrm{W} 49 \mathrm{~N}$ form in the outer shock of a protostellar jet cocoon retains the observationally verified part of the previous proposal by Mac Low \& Elitzur (1992): the masers trace the surface of an elongated shell, expanding quickly at the ends and slowly along the sides. 
At the same time, it resolves the aspect of the earlier proposal that seemed most difficult to explain. We no longer require a large cylindrical cavity surrounding the protostar to collimate a shell driven by a spherically symmetric wind. Instead, the collimation is an inherent property of the outflow and can occur much closer to the star, as suggested by current jet models (e.g., Königl \& Ruden 1993). Furthermore, we can now explain the clumping of the masers observed by Gwinn et al. (1994) as dynamical instabilities in the dense shell surrounding the cocoon.

A jet-driven cocoon will produce water masers whenever the right combination of conditions occurs on its surface-an ambient density $\gtrsim 3 \times 10^{6} \mathrm{~cm}^{-3}$ and a shock velocity $\sim 20-$ $200 \mathrm{~km} \mathrm{~s}^{-1}$ (Elitzur et al. 1989; Kaufman \& Neufeld 1993). In W49N it appears that the entire shell currently meets these requirements. As the cocoon expands, the masers will turn off because of two effects. First, the ambient density is likely to decrease with distance from the central source and fall below the lower bound for maser production. Second, since $v_{h}$ increases with decreasing $n_{a}$, the cooling parameter for the Mach disk is also expected to decrease with time; once $\chi$ drops below unity, the interior of the cocoon begins to cool and can no longer drive strong shocks out to the side. Either one of these mechanisms could change the appearance of the source quite rapidly, which suggests that the elongated shell structure observed in $\mathrm{W} 49 \mathrm{~N}$ is unlikely to be detected in most other maser sources.

As the jet sweeps up more gas, it will eventually be observable as a bipolar molecular outflow. Another star in the W49N complex has apparently already evolved to this stage. In radio continuum radiation, a bipolar ionized structure has been mapped by Welch et al. (1987), as seen on the far right of their Figure 4, while a bipolar $\mathrm{CO}$ outflow in the region was mapped by Scoville et al. (1986) at somewhat lower resolution. Felli, Palagi, \& Tofani (1992) have shown that water masers are associated with $\mathrm{CO}$ outflows, and that the maser luminosity is correlated with the mechanical luminosity of the outflow. Although the full shell will no longer produce maser emission, the tip of the jet may as long as it encounters dense enough material.

Magnetic fields could in principle explain a cylindrical confinement of a spherically symmetric expansion. However, masers are observed in W49N with velocities as high as $200 \mathrm{~km}$ $\mathrm{s}^{-1}$. Thus, the magnetic field would need to collimate a spherically expanding shell with an expansion velocity of $v_{e} \sim 200$ $\mathrm{km} \mathrm{s}^{-1}$. To find the field strength required, $B_{\text {coll }}$, we can use equation (10), with the same ambient density, but substituting $v_{e}$ for $v_{c}$ to find $B_{\text {coll }} \sim 0.3 \mathrm{G}$. Such large fields in the ambient gas are inconsistent with the observation of $50 \mathrm{mG}$ fields in the postshock maser clumps by Fiebig \& Güsten (1989), as discussed in the previous section.

$\mathrm{W} 49 \mathrm{~N}$ is the only water maser source studied at sufficient detail to enable an inference of its kinematic and geometric structure. However, it is not a typical source. With a maser isotropic luminosity of $\sim 1 L_{\odot}$, it is about five orders of magnitude more luminous than common Galactic water maser sources (Felli et al. 1992). From an analysis that incorporated observations of nearby galaxies, Greenhill et al. (1990) concluded that, on average, a galaxy contains only one source of the luminosity class of W49N. However, like the other stars in this association, the protostar driving the jet in $\mathrm{W} 49 \mathrm{~N}$ is probably extremely massive and may have formed under unusual circumstances (see Serabyn, Güsten, \& Schulz 1993). The W49N cocoon has expanded to its present size in only $\sim 350$ yr. Its uniqueness is thus probably due to the combination of the lifetime of the phase and the existence of only $\sim 10^{4} \mathrm{O}$ stars in the Galaxy (Wood \& Churchwell 1989). This source thus provides a unique opportunity for a detailed study of jet-driven cocoons.

We thank M. L. Norman for pointing out the applicability of his computations to this problem, and C. Gwinn for discussing his results with us. M.-M. M. L. and A. K. were partially supported by NASA grant NAGW-2379 and M. E. by NSF grant AST-9016810. Computations were performed at the National Center for Supercomputing Applications.
Begelman, M. C., \& Cioffi, D. F. 1989, ApJ, 345, L21

Blondin, J. M., Fryxell, B. A., \& Königl, A. 1990, ApJ, 360, 370

Cioffi, D. F., \& Blondin, J. M. 1992, ApJ, 392, 458

Colella, P., \& Woodward, P. R. 1984, J. Comput. Phys., 54, 174

de Gouveia Dal Pino, E. M., \& Benz, W. 1993, ApJ, 410, 686

Elitzur, M., Hollenbach, D. J., \& McKee, C. F. 1989, ApJ, 346, 983

Felli, M., Palagi, F., \& Tofani, G. 1992, A\&A, 255, 293

Fiebig, D., \& Güsten, R. 1989, A\&A, 214, 333

Greenhill, L. J., et al. 1990, ApJ, 364, 513

Gwinn, C. R. 1993, in Ástrophysical Masers, ed. A. W. Clegg \& G. E. Nedoluha (Berlin: Springer), 241

Gwinn, C. R., Moran, J. M., \& Reid, M. J. 1992, ApJ, 393, 149 1994, ApJ, submitted

Kaufman, M. J., \& Neufeld, D. A. 1994, in preparation

Königl, A. 1982, ApJ, 261, 115

Königl, A., \& Ruden, S. P. 1993, in Protostars \& Planets III, ed. E. H. Levy \&

J. I. Lunine (Tucson: Univ. of Arizona Press), 641

\section{REFERENCES}

Loken, C., Burns, J. O., Clarke, D. A., \& Norman, M. L. 1992, ApJ, 392, 54

Mac Low, M.-M., \& Elitzur, M. 1992, ApJ, 393, L33 (Paper I)

Mac Low, M.-M., \& Norman, M. L. 1993, ApJ, 407, 207

Masson, C. R., \& Chernin, L. M. 1993, ApJ, 414, 230

Norman, M. L., Smarr, L., Winkler, K.-H. A., \& Smith, M. D. 1982, A\&A, 113, 285

Scheuer, P. A. G. 1974, MNRAS, 166, 513

Scoville, N. X., Sargent, A. I., Sanders, D. B., Claussen, M. J., Masson, C. R., Lo, K. Y., \& Phillips, T. G. 1986, ApJ, 303, 416

Serabyn, E., Güsten, R., \& Schulz, A. 1993, ApJ, 413, 571

Stone, J. M., \& Norman, M. L. 1993a, ApJ, 413, 198

$$
\text { . 1993b, ApJ, 413, } 210
$$
1994, ApJ, 420, 237

Vishniac, E. T. 1983, ApJ, 274, 152

Welch, W. J., Dreher, J. W., Jackson, J. M., Terebey, S., \& Vogel, S. N. 1987, Science, 238, 1550

Wood, D. O.S., \& Churchwell, E. 1989, ApJ, 340, 265 